\title{
On Two Alternative Uniformly Asymptotic Procedures for Analyzing the High Frequency Diffraction of a Complex Source Beam by a Straight Wedge
}

\author{
Hsi-Tseng Chou, Prabhakar H. Pathak, Youngchel Kim and Giuliano Manara
}

\begin{abstract}
When spectral wave integrals, representing the radiation and diffraction of electromagnetic (EM) waves, are characterized by a first order saddle point and poles in the integrand, they can usually be evaluated, in essentially closed form, at high frequencies by the leading terms of any of the two wellknown alternative uniformly asymptotic procedures, namely the Pauli - Clemmow method (PCM) and the Van der Waerden method (VWM), respectively. The PCM has the advantage that its leading terms directly yield a solution in the simple ray format of the uniform geometrical theory of diffraction (UTD). On the other hand, it is commonly noted that it is not the leading terms of the PCM but those of the VWM which remain valid for the case of complex waves. Nevertheless, it is shown here that the PCM can surprisingly work even for some special complex wave cases, only if certain conditions are met. Indeed, it is demonstrated here that the PCM meets these conditions for the special case of the diffraction of a complex source beam (CSB) by a wedge. Also, the PCM directly yields a UTD like solution for this case. The latter result is significant as it provides a strong justification for obtaining a simple UTD type solution for the more general problem of the diffraction of a CSB incident from an arbitrary direction on a wedge with arbitrary curvature, directly via analytic continuation of the corresponding UTD result available for a curved wedge illuminated by a point source in real space. It is also shown that the VWM solution can be trivially cast in the UTD format, by expressing it as a sum of the PCM solution plus a UTD like correction term; a similar result was obtained previously using a higher order term from a generalization of the PCM procedure given elsewhere.
\end{abstract}

\section{INTRODUCTION}

$\mathrm{T}$ he present work investigates the behavior and validity of the leading terms of two different uniform asymptotic high frequency procedures, namely the PCM and the VWM, respectively, for analyzing the canonical problem of EM diffraction of a CSB field when it is incident on a perfectly

Manuscript received on Oct. 9, 2017

This work was financially sponsored by the Ministry of Science and Technology, Taiwan.

H.-T. Chou is with the Graduate Institute of Communication Engineering, National Taiwan University, Taipei 10617, Taiwan (e-mail: chouht@ntu.edu.tw)

P. H. Pathak is with ECE dept., the Ohio State Univ., Columbus, OH43212, USA, and EE dept., Univ. of South Florida, Tampa, Florida (e-mail: pathakph@gmail.com).

Y. Kim, deceased, was formerly with the Ohio State Univ., Columbus, OH43212, and most recently at Lockheed Martin, Denver, Colorado, USA

G. Manara is with Department of Information Engineering, University of Pisa, 56122-Pisa, Italy (e-mail: g.manara@,iet.unipi.it). conducting straight wedge. The motivation for this work is as follows.

Formal solutions to EM canonical radiation and diffraction problems are often expressed in terms of spectral integrals which rarely lend themselves to an exact solution in analytical closed form. However, at high frequencies, they can be evaluated in essentially closed form (involving special functions characteristic of the radiation/diffraction process), using available uniformly asymptotic procedures. Such asymptotic approximations typically provide a simple physical picture for the wave radiation and diffraction mechanisms. When spectral wave integrals are characterized by a first order saddle point and poles in the integrand, they can be evaluated via two of the well-known, alternative, uniformly asymptotic methods, namely the PCM [1]-[4] and the VWM [4]-[7], respectively. In the PCM, and the VWM, the original complex contour of integration over the spectral variable is deformed into the steepest descent path (SDP) through the saddle point in the integrand, with a possibility that a pole of the integrand may be captured in this contour deformation. The pole typically migrates when the observation point moves, and in so doing it can cross the SDP either through the saddle point as it usually does for real waves, or away from it as in the case of complex wave problems, respectively. Some common examples of complex waves include complex source beams (CSBs), surface and leaky waves, and other types of evanescent waves. Also, it is known that a CSB is produced by a point source which is positioned in complex space [10]-[12]. Now, the PCM has the advantage that it directly yields a solution in the simple ray format of the uniform geometrical theory of diffraction (UTD) [3],[8],[9]. On the other hand, it is commonly noted that it is not the PCM but the VWM which remains valid if the pole crosses the SDP away from the saddle point [4], as it usually does in the case of complex wave problems. Nevertheless, it is shown here that the PCM can work surprisingly well even in such latter situations, if certain conditions are met. Indeed, it is demonstrated here that the PCM meets these conditions for the special case of diffraction of a CSB by a straight wedge which is studied in this paper; thus, the PCM is shown to have essentially the same accuracy as the VWM in this case. Furthermore, the PCM also directly yields a UTD like solution for this case. The latter result is quite significant since it provides a justification for obtaining a physically appealing UTD type solution, in a direct manner, for the more general 
problem of the diffraction of a CSB incident from an arbitrary direction on a wedge with arbitrary curvature, simply via analytic continuation of the corresponding UTD solution available in [8] for a curved wedge illuminated by a point source in real space. Due to space limitations, the latter direct generalization to analytically describe the fields around an arbitrary curved wedge excited by a CSB, and also to the case when it is excited by an astigmatic Gaussian beam (GB), will be described with applications in detail in a separate paper.

Some previous publications related to the PCM and VWM approaches may be found in [13]-[20]. In [15], a complete PCM based expansion is given which is valid for many simple poles coalescing with the saddle point. It is noted that calculating higher order terms to obtain a complete expansion, beyond just the leading terms in the saddle point approximation is tedious, and needs considerable effort [15]. In [16], the VWM treatment of [6] is generalized to include many simple poles in the spectral integrand, but only leading terms are retained. An attempt to relate PCM to VWM is described in [17]. It is analytically demonstrated that a full equivalence between the PCM and VWM can be established only if all terms of the complete expansion are retained in both methods [18]. A development similar to [18] is presented for the straight edge geometries in [19],[20] excited by a real source. In [6], the uniform nature of the VWM is made possible through the presence of an error function, whereas that in [4] includes a Fresnel type function instead, for both PCM and VWM. The latter Fresnel type function is the same as that present in the available UTD for wedges excited by sources in real space [3]. It is noted that only the VWM has been applied previously to treat the problem of CSB diffraction by a canonical perfectly conducting straight wedge [21], but that solution was not expressed directly in the UTD like format. A PCM solution was obtained for the diffraction of an inhomogeneous type complex plane wave by a conducting wedge in which the next higher order term beyond the usual leading terms had to be retained, to make the PCM solution work for this case [13],[14]. In this paper, the leading terms of both PCM and the VWM are obtained and compared for the case of a wedge excited by a CSB, and it is shown that the PCM surprisingly remains valid for this special case even though in general it is not valid for complex waves.

The PCM and VWM based leading terms are presented here in a complete fashion for application. In this regard, it is noted that the PCM as described in an Appendix of [4] is expressed in the format of the Sommerfeld half plane type solution which is not in UTD form, while the VWM in [4] is also not in UTD like form. Here, the VWM solution is expressed as a PCM solution, which has a UTD form, plus a UTD like correction term. Thus, if a PCM solution fails to be uniform, then the latter UTD like correction can be simply added to restore the uniform property; a similar result was obtained earlier in [13],[14] using a higher order term from a generalized PCM solution developed in [15].

The format of the present paper is as follows. Section II summarizes the development of the leading terms of the PCM and VWM methods. The nature of the pole wave discontinuity and its compensation is discussed for both the PCM and the VWM in section III. It is seen that the leading terms of the PCM will work as well as those of the VWM for the complex wave case only if certain conditions are met. In section IV it is shown that the above conditions are met in the development of the solution to the canonical problem of diffraction of a CSB by a straight conducting wedge; in this special case it is shown analytically that the difference between the PCM and VWM is essentially negligible. While the authors have analyzed both, the two and three dimensional problems of complex line and point source illumination of the wedge, only the line source case is discussed in this paper for the sake of simplicity. Numerical results for the CSB diffraction by a wedge obtained here by the PCM and VWM are also seen to compare quite well. Some conclusions are given in section V. An $e^{+j \omega t}$ time convention for the source and fields is assumed and suppressed in the following.

\section{DEVELOPMENT OF THE LEADING TERMS IN THE PCM AND VWM}

Consider a typical spectral radiation/diffraction integral, $I(K)$ given by:

$$
I(K)=\int_{C} g(\xi) e^{K f(\xi)} d \xi,
$$

where $K$ is a real parameter here, and $C$ is a complex contour on which $I(K)$ converges. Generally, $I(K)$ is not amenable to an exact analytical solution in closed form. However, $I(\mathrm{~K})$ can be approximated in essentially closed form by the leading terms of an asymptotic expansion for large $K$ [6]. If one assumes that $f(\xi)$ exhibits a first order saddle point and $g(\xi)$ has a simple pole (although the methods below can be modified to include many poles including higher order poles), then the PCM and VWM which are based on a saddle point technique can be employed to evaluate $I(K)$. The first order saddle point at $\xi=\xi_{s}$ satisfies:

$$
\left.\frac{d f}{d \xi}\right|_{\xi=\xi_{s}}=f^{\prime}\left(\xi_{s}\right)=0 ; f^{\prime \prime}\left(\xi_{s}\right) \neq 0,
$$

where primes in (2) denote derivatives with respect to $\xi$. Let a simple pole of $g(\xi)$ exist at $\xi=\xi_{p}$. The PCM and VWM begin by deforming the original contour $C$ into a steepest decent path (SDP) through $\xi_{\mathrm{s}}$ [6]. It is noted that on the SDP, $\operatorname{Re} f(\xi) \leq \operatorname{Re} f\left(\xi_{s}\right)$ and $\operatorname{Im} f(\xi)=\operatorname{Im} f\left(\xi_{s}\right)$ where Re and $\operatorname{Im}$ denote the real and imaginary parts. An application of the Cauchy residue theorem yields [6]:

$$
I(K)=\int_{C} g(\xi) e^{K f(\xi)} d \xi=\left[\int_{S D P} g(\xi) e^{K f(\xi)} d \xi+2 \pi j R_{p} \Lambda U\right],
$$

where it is assumed that no branch cuts are crossed in deforming C to SDP. Here, $R_{p}$ is the residue of the simple pole at $\xi=\xi_{p}$, namely,

$$
R_{p}=\lim _{\xi \rightarrow \xi_{p}}\left(\xi-\xi_{p}\right) g(\xi) e^{K f(\xi)},
$$

or

$$
R_{p} e^{-K f\left(\xi_{p}\right)}=\lim _{\xi \rightarrow \xi_{p}}\left(\xi-\xi_{p}\right) g(\xi) .
$$

The $\Lambda=( \pm 1)$, when [C-SDP] encloses the pole at $\xi_{p}$ in a ( counter clockwise $(\mathrm{CCW})$ clockwise (CW) sense. Also, the step function 
$U \equiv\left[\begin{array}{l}1, \text { if } \xi_{p} \text { is enclosed by (C-SDP) } \\ 0, \text { otherwise }\end{array}\right]$. On the SDP, one notes that the phase of $e^{K f(\xi)}$ is a constant $\left(\operatorname{Im} f(\xi)=\operatorname{Im} f\left(\xi_{s}\right)\right)$, so besides the pole of $g(\xi)$ at $\xi_{p}$, the exponential $e^{K \operatorname{Re} f(\xi)}$ controls the topology of the integrand in the vicinity of the SDP. The dominant contribution to the SDP integral arises from $\xi=\xi_{s}$; however, the presence of $\xi_{p}$ can affect the saddle point contribution if $\xi_{p}$ lies in vicinity of $\xi_{s}$. The influence of a nearby $\xi_{p}$ on the contribution from $\xi=\xi_{s}$ is accounted by a uniform transition function, $F(\cdot)$, in PCM and VWM as will be evident later. The following transformation maps the SDP onto a real axis in a complex $\alpha$ plane [6]

$$
f(\xi)=f\left(\xi_{\mathrm{s}}\right)-\alpha^{2} .
$$

The $\xi_{s}$ maps to $\alpha=0$; also, $\xi_{p}$ maps to $\alpha=\alpha_{p}$. Thus, (5) yields

$$
I_{S D P}(K)=\int_{S D P} g(\xi) e^{K f(\xi)} d \xi=\int_{-\infty}^{\infty} g(\xi) \frac{d \xi}{d \alpha} e^{K\left[f\left(\xi_{s}\right)-\alpha^{2}\right]} d \alpha,
$$

or

$$
I_{S D P}(K)=e^{K f\left(\xi_{s}\right)} \int_{-\infty}^{\infty} G(\alpha) e^{-K \alpha^{2}} d \alpha ; G(\alpha) \equiv g(\xi) \frac{d \xi}{d \alpha} .
$$

For $\xi$ near $\xi_{\text {s }}$ (i.e. for $\alpha$ near $\alpha=0$ ),

$$
f(\xi) \approx f\left(\xi_{s}\right)+\frac{f^{\prime \prime}\left(\xi_{s}\right)}{2}\left(\xi-\xi_{s}\right)^{2} \text { or } \alpha^{2} \approx-\frac{f^{\prime \prime}\left(\xi_{s}\right)}{2}\left(\xi-\xi_{s}\right)^{2} .
$$

Also, differentiating (5) twice with respect to $\alpha$ yields

$$
\left.\frac{d \xi}{d \alpha}\right|_{\xi=\xi_{s}}=\sqrt{\frac{-2}{f^{\prime \prime}\left(\xi_{s}\right)}} \equiv\left|\sqrt{\frac{-2}{f^{\prime \prime}\left(\xi_{s}\right)}}\right| e^{j \phi_{s}},
$$

where the branch of the square root is chosen so that $\phi_{\mathrm{s}}=\arg (d \xi)$ along the descending part of the SDP [6]. From (6) and (7), $G(\alpha)$ at $\alpha=0$ is given by

$$
G(0)=g\left(\xi_{s}\right)\left|\sqrt{\frac{-2}{f^{\prime \prime}\left(\xi_{s}\right)}}\right| e^{j \phi_{s}} .
$$

A measure of separation between $\xi_{p}$ and $\xi_{s}$; i.e., and hence between $\alpha_{p}$ and $\alpha_{s}=0$, is defined in terms of a parameter " $a$ " as follows:

$$
f\left(\xi_{s}\right)-f\left(\xi_{p}\right)=\alpha_{p}^{2} \equiv-j a .
$$

Next, the $I(K)$ of (3) will be evaluated by PCM in part A, and by VWM in part B, below.

\section{A. The PCM Development}

In the PCM, the pole at $\alpha=\alpha_{p}$ in $G(\alpha)$ is split off as a factor, namely [1]-[3],[19]

$$
G(\alpha) \equiv \frac{G_{a}(\alpha)}{\alpha-\alpha_{p}}
$$

where $G_{a}(\alpha)$ is analytic in the $\alpha$ plane. From (3)

$$
I^{P C M}(K)=2 \pi j R_{p} \Lambda U+I_{S D P}^{P C M},
$$

where the superscript PCM on $I(K)$ and $I_{S D P}$ denotes that the latter are evaluated in this subsection via PCM, and from (6)

$$
I_{S D P}^{P C M}(K)=e^{K f\left(\xi_{s}\right)} \int_{-\infty}^{\infty} \frac{G_{a}(\alpha)}{\alpha-\alpha_{p}} e^{-K \alpha^{2}} d \alpha .
$$

Since $G_{a}(\alpha)$ is analytic near $\alpha=0$, one may expand $G_{a}(\alpha)$ in a Taylor series as $G_{a}(\alpha)=\sum_{n=0}^{\infty} c_{n} \alpha^{n}$. Retaining only $n=0$ term yields $G_{a}(\alpha) \approx G_{a}(0)$; thus (12) becomes

$$
I_{S D P}^{P C M}(K) \sim e^{K f\left(\xi_{s}\right)} G_{a}(0) \alpha_{p} \int_{-\infty}^{\infty} \frac{e^{-K \alpha^{2}}}{\alpha^{2}-\alpha_{p}^{2}} d \alpha .
$$

From (8) and (10), one writes (13) as

$$
\begin{aligned}
& I_{S D P}^{P C M}(K) \sim-\alpha_{p}^{2} e^{K f\left(\xi_{s}\right)} G(0) \int_{-\infty}^{\infty} \frac{e^{-K \alpha^{2}}}{\alpha^{2}-\alpha_{p}^{2}} d \alpha \\
& =e^{K f\left(\xi_{s}\right)} j a g\left(\xi_{s}\right)\left|\sqrt{\frac{-2}{f "\left(\xi_{s}\right)}}\right| e^{j \phi_{s}} \int_{-\infty}^{\infty} \frac{e^{-K \alpha^{2}}}{\alpha^{2}-\alpha_{p}^{2}} d \alpha
\end{aligned}
$$

since $G_{a}(0)=-\alpha_{p} G(0)$, and $\alpha_{p}^{2}=-j a$ via (9). Further rearrangement of (14) yields

$I_{S D P}^{P C M}(K) \sim g\left(\xi_{s}\right)\left|\sqrt{\frac{-2 \pi}{K f^{\prime \prime}\left(\xi_{s}\right)}}\right| e^{j \phi_{s}} e^{K f\left(\xi_{s}\right)} \cdot\left[j a \sqrt{\frac{K}{\pi}} \int_{-\infty}^{\infty} \frac{e^{-K \alpha^{2}}}{\alpha^{2}-\alpha_{p}^{2}} d \alpha\right]$

or

$I_{S D P}^{P C M}(K) \sim g\left(\xi_{s}\right)\left|\sqrt{\frac{-2 \pi}{K f "\left(\xi_{s}\right)}}\right| e^{j \phi_{s}} e^{K f\left(\xi_{s}\right)} F( \pm \sqrt{K a}) ; \operatorname{Im} \alpha_{p}{ }_{>}^{<}$,

where, from the Appendix, the uniform transition function $F$ is:

$$
F( \pm \sqrt{K a}) \equiv j a \sqrt{\frac{K}{\pi}} \int_{-\infty}^{\infty} \frac{e^{-K \alpha^{2}}}{\alpha^{2}-\alpha_{p}^{2}} d \alpha ; \operatorname{Im} \alpha_{p}<0,
$$

or

$$
F( \pm \sqrt{K a}) \equiv 2 j( \pm \sqrt{K a}) e^{j K a} \int_{ \pm \sqrt{K a}}^{\infty} e^{-j \tau^{2}} d \tau ; \operatorname{Im} \alpha_{p}<0 .
$$

One may rewrite (15) as

$$
I_{S D P}^{P C M}(K) \sim\left(I_{S D P}^{N U}\right) F( \pm \sqrt{K a}),
$$

where

$$
I_{S D P}^{N U}(K) \equiv g\left(\xi_{s}\right)\left|\sqrt{\frac{-2 \pi}{K f^{\prime \prime}\left(\xi_{s}\right)}}\right| e^{j \phi_{s}} e^{K f\left(\xi_{s}\right)} .
$$

If $| \pm \sqrt{K a}| \rightarrow \infty$ then $F( \pm \sqrt{K a}) \rightarrow 1 \quad$ (see Appendix) and $I_{S D P}^{P C M} \rightarrow I_{S D P}^{N U}$. It is noted that $F( \pm \sqrt{K a})$ is valid for $\left(\begin{array}{c}-3 \pi / 4<\arg \sqrt{K a}<\pi / 4 \\ \pi / 4<\arg \sqrt{K a}<5 \pi / 4\end{array}\right)$, i.e., when $\operatorname{Im} \alpha_{p}{ }_{>} 0$, since $\alpha_{p}^{2}=-j a\left(\right.$ or $\left.a=e^{j \pi / 2} \alpha_{p}^{2}\right)$, or $\sqrt{K a}=\alpha_{p} e^{j \pi / 4} \sqrt{K}$, which implies that $\arg \sqrt{K a}=\left(\arg \alpha_{p}\right)+\pi / 4$. The $I^{P C M}(K)$ of (11) together with the asymptotic result in $(15 b)$ completes the development for the leading terms of PCM.

\section{B. The VWM Approach}

In the VWM, the pole at $\alpha=\alpha_{p}$ in $G(\alpha)$ is split off in an additive fashion, namely [4]-[7]

$$
G(\alpha)=H_{a}(\alpha)+\frac{R_{p} e^{-K f\left(\xi_{p}\right)}}{\alpha-\alpha_{p}} \quad,
$$

and it is clear that $H_{a}(\alpha)$ is analytic near $\alpha=0$ and its 
neighborhood. Note that $R_{p} e^{-K f\left(\xi_{p}\right)}=\lim _{\alpha \rightarrow \alpha_{p}}\left(\alpha-\alpha_{p}\right) G(\alpha)$ $=\lim _{\alpha \rightarrow \alpha_{p}}\left(\xi-\xi_{p}\right) g(\xi)$ as before in (4). Thus, from (3)

$$
I^{V W M}(K)=2 \pi j R_{p} \Lambda U+I_{S D P}^{V W M}(K),
$$

where the superscript VWM on $I(K)$ and $I_{S D P}(K)$ denotes the latter are being evaluated in this subsection via VWM. From (6) and (19)

$$
I_{S D P}^{V W M}(K)=e^{K f\left(\xi_{s}\right)}\left[\int_{-\infty}^{\infty} H_{a}(\alpha) e^{-K \alpha^{2}} d \alpha+R_{p} e^{-K f\left(\xi_{p}\right)} \alpha_{p} \int_{-\infty}^{\infty} \frac{e^{-K \alpha^{2}}}{\alpha^{2}-\alpha_{p}^{2}} d \alpha\right]
$$

Since $H_{a}(\alpha)$ is analytic at and near $\alpha=0$, one may use a Taylor expansion $H_{a}(\alpha)=\sum_{n=0}^{\infty} a_{n} \alpha^{n}$, and retain only the leading term $(n=0)$ with $a_{0}=H_{a}(0)$ as

$$
\begin{aligned}
& I_{S D P}^{V W M} \sim e^{K f\left(\xi_{s}\right)}\left[G(0)+\frac{R_{p} e^{-K f\left(\xi_{p}\right)}}{\alpha_{p}}\right] \int_{-\infty}^{\infty} e^{-K \alpha^{2}} d \alpha \\
& +R_{p} e^{K \alpha_{p}^{2}} \alpha_{p} \int_{-\infty}^{\infty} \frac{e^{-K \alpha^{2}}}{\alpha^{2}-\alpha_{p}^{2}} d \alpha
\end{aligned}
$$

Since $\quad f\left(\xi_{s}\right)-f\left(\xi_{p}\right)=-j a=\alpha_{p}^{2} \quad$ via $\quad$ (9), and $H_{a}(0)=G(0)+\frac{R_{p} e^{-K f\left(\xi_{p}\right)}}{\alpha_{p}}$ via (19). Also, $\int_{-\infty}^{\infty} e^{-K \alpha^{2}} d \alpha=\sqrt{\frac{\pi}{K}}$. Thus

$$
I_{S D P}^{V W M}(K)=I_{S D P}^{N U}(K)+\frac{R_{p} e^{-j K a}}{\sqrt{-j a}} \sqrt{\frac{\pi}{K}}[1-F( \pm \sqrt{K a})],
$$

where (9), (16) and (18) have been utilized in (22). The leading terms of $I^{V W M}(K)$ is given by the expression in (23), thus completing the VCM development for the leading terms.

\section{UNIFORM BEHAVIOR OF THE LEADING TERMS OF PCM AND VWM AND THEIR RELATIONSHIP}

An analytical study of the uniform behavior of the PCM and VWM, of (11) and (20), is conducted below. The PCM is studied first in part A, and the VWM is studied next in part B. A relationship between the leading terms of the VWM and PCM is simply established in part $\mathrm{C}$ as

$$
I^{V W M}(K)=I^{P C M}(K)+E(K),
$$

where $E(K)$ is a correction to PCM for complex waves and is obtained in a trivial fashion by simply taking the difference between (20) and (11), respectively and rearranging terms. The conditions under which $E(K) \rightarrow 0$ are also described in part C. It is shown in section IV that $E \approx 0$ for the problem of CSB diffraction by a straight wedge, which is of special interest in this paper.

The complete leading terms of PCM and VWM are summarized explicitly below for convenience so their properties and relationship can be studied. From (11) and (17)

$$
I^{P C M}(K) \sim 2 \pi j R_{p} \Lambda U+I_{S D P}^{N U}(K) F( \pm \sqrt{K a}) ; \operatorname{Im} \alpha_{p}{ }_{>}^{<} 0,
$$

and likewise from (20) and (23):

$$
\begin{aligned}
& I^{V W M}(K) \sim 2 \pi j R_{p} \Lambda U+I_{S D P}^{N U}(K) \\
& +\frac{R_{p} e^{-j K a}}{\sqrt{-j a}} \sqrt{\frac{\pi}{K}}[1-F( \pm \sqrt{K a})] ; \operatorname{Im} \alpha_{p}<0 .
\end{aligned}
$$

The uniform nature of PCM and VWM is supposed to provide continuity of $I^{P C M}(K)$ and $I^{V W M}(K)$, respectively, as the pole at $\alpha=\alpha_{p}$ crosses the $\operatorname{SDP}$ (along $-\infty<\operatorname{Re} \alpha<\infty$ ).

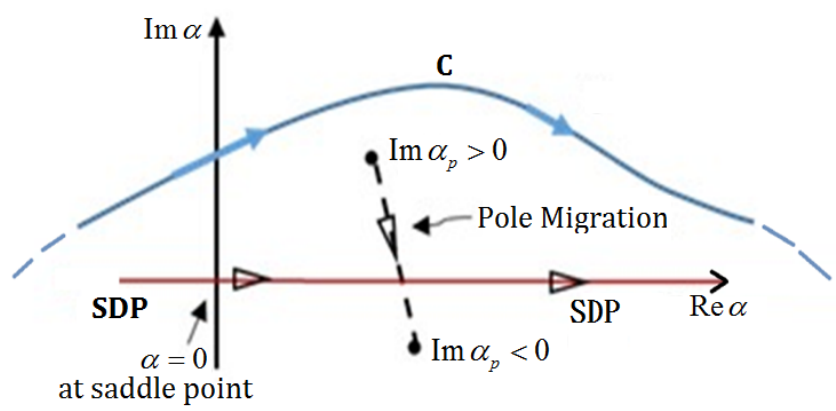

(a) Case (a)

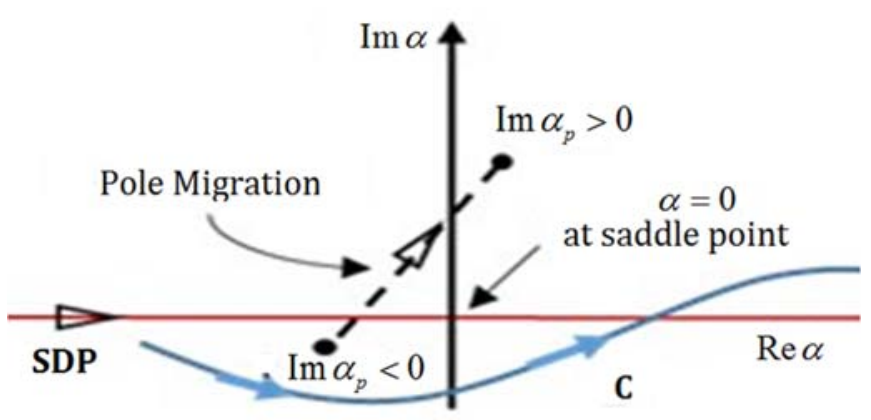

(b) Case (b)

Fig 1 : Migration of the pole at $\alpha=\alpha_{p}$ across the $\operatorname{SDP}($ or $\operatorname{Im} \alpha=0$ )

Case (a) : pole $\alpha_{p}$ moves from the region $\operatorname{Im} \alpha_{p}>0$ to $\operatorname{Im} \alpha_{p}<0$ or vice versa, and with the contour $\mathrm{C}$ shown for pole capture when $\operatorname{Im} \alpha_{p}>0$.

Case (b): pole $\alpha_{p}$ mores from the region $\operatorname{Im} \alpha_{p}<0$ to $\operatorname{Im} \alpha_{p}>0$ or vice versa, and with a different contour $\mathrm{C}$ as shown for pole capture when $\operatorname{Im} \alpha_{p}<0$

\section{A. Uniform Behavior of PCM}

For case (a) of Fig. 1, where the pole at $\alpha=\alpha_{p}$ is initially in the region $\operatorname{Im} \alpha_{p}>0$, so that it is captured in a clockwise $(\mathrm{CW})$ sense in deforming $\mathrm{C}$ into SDP in this situation; thus $\Lambda=-1$ and $U=1$ here, consequently, from (25):

$$
\left.I^{P C M}(K)\right|_{\operatorname{Im} \alpha_{p}>0} \equiv I_{>}^{P C M}(K) \sim 2 \pi j\left(-R_{p}\right)+I_{S D P}^{N U}(K) F(-\sqrt{K a}),
$$

where $F(-\sqrt{K a})$ is chosen in (27) since $\operatorname{Im} \alpha_{p}>0$. Next, when the pole at $\alpha=\alpha_{p}$ now crosses the $\operatorname{SDP}$ (on the $\operatorname{Re} \alpha$ axis) as it moves from the region $\operatorname{Im} \alpha_{p}>0$ to the region $\operatorname{Im} \alpha_{p}<0$, then in the region $\operatorname{Im} \alpha_{p}<0$ a pole is no longer captured upon deforming $\mathrm{C}$ into SDP. Hence, for $\operatorname{Im} \alpha_{p}<0$, $U=0$, so that from (25): 


$$
\left.I^{P C M}(K)\right|_{\operatorname{Im} \alpha_{p}<0} \equiv I_{<}^{P C M}(K) \sim 0+I_{S D P}^{N U}(K) F(+\sqrt{K a}),
$$

where $F(+\sqrt{K a})$ is chosen in (28) since $\operatorname{Im} \alpha_{p}<0$. Now the continuity of $I^{P C M}$ as $\alpha_{p}$ just crosses the SDP requires that

$$
\lim _{\operatorname{Im} \alpha_{p} \rightarrow 0}\left(I_{>}^{P C M}(K)-I_{<}^{P C M}(K)\right)=0 .
$$

From (27) and (28), it is clear that (29) is true if

$$
\lim _{\operatorname{Im} \alpha_{p} \rightarrow 0}\left(I_{S D P}^{N U}(K)[F(-\sqrt{K a})-F(+\sqrt{K a})]\right)=2 \pi j R_{p} .
$$

From (9), (10), (18) and (A-6), one may check if (30) is true, i.e.,

or

$$
G_{a}(0) \frac{e^{K f\left(\xi_{p}\right)}}{-\sqrt{-j a}}(-2 \pi \sqrt{j a})=2 \pi j R_{p},
$$

$$
G_{a}(0) e^{K f\left(\xi_{p}\right)}=G_{a}\left(\alpha_{p}\right) e^{K f\left(\xi_{p}\right)} .
$$

In (32), one employs the relation $R_{p}=G_{a}\left(\alpha_{p}\right) e^{K f\left(\xi_{p}\right)}$ via (4) and $\lim _{\xi \rightarrow \xi_{p}}\left(\xi-\xi_{p}\right) g(\xi)=\lim _{\alpha \rightarrow \alpha_{p}}\left(\alpha-\alpha_{p}\right) G(\alpha)$. From (10), $G_{a}(\alpha)=\left(\alpha-\alpha_{p}\right) G(\alpha)$ so that $\lim _{\xi \rightarrow \xi_{p}}\left(\xi-\xi_{p}\right) g(\xi)=\lim _{\alpha \rightarrow \alpha_{p}} G_{a}(\alpha)$ $=G_{a}\left(\alpha_{p}\right)$, which leads to $R_{p} e^{-K f\left(\xi_{p}\right)}=G_{a}\left(\alpha_{p}\right)$. Clearly, (32) is satisfied only if $G_{a}(0)=G_{a}\left(\alpha_{p}\right)$, or $\alpha_{p}=0$ when $\operatorname{Im} \alpha_{p} \rightarrow 0$; i.e., if $\alpha_{p}$ crosses the SDP exactly through the saddle point (at $\alpha=0$ ). However, one can obviously summarize the conditions under which $I^{P C M}(K)$ is uniform or continuous in an approximate manner such that the discontinuity becomes negligible in a practical sense, namely if:

(i) $G_{a}(0) \cong G_{a}\left(\alpha_{p}\right)$ as $\operatorname{Im} \alpha_{p} \rightarrow 0$, even if $\alpha_{p}$ does not cross the SDP through $\alpha_{s}$ (or $\left.\alpha \equiv \alpha_{s}=0\right)$.

(ii) $G_{a}(\alpha)$ is almost constant or slowly varying in the neighborhood of and including the SDP (along $\operatorname{Im} \alpha=0$ ) so that $G_{a}^{\prime}(\alpha) \approx 0$ along the SDP.

Also, if $G_{a}(0)=G_{a}\left(\alpha_{p}\right)$ on the SDP, or if $G_{a}^{\prime}(\alpha)=0$ then the PCM is uniformly continuous additionally, as pointed via (32), if $G_{a}\left(\alpha_{p}\right)=G_{a}(0)$ when $\alpha_{p}$ crosses the SDP through the saddle point ( $\alpha=0)$, then the PCM is of course continuous. One can likewise conclude the same result if one were to check the continuity of $I^{P C M}(K)$ for case (b) of Fig. 1, where $\Lambda=+1$ and $U=1$ in $I_{<}^{P C M}(K)$ for $\operatorname{Im} \alpha_{p}<0$, while $U=0$ in $I_{>}^{P C M}(K)$ for $\operatorname{Im} \alpha_{p}>0$. Then, following the above procedure, continuity of $I^{P C M}(K) \quad$ requires $\lim _{\operatorname{Im} \alpha_{p} \rightarrow 0}\left[I_{>}^{P C M}(K)-I_{<}^{P C M}(K)\right]=0$, which again leads exactly to the same conclusion as above (for case (a)).

All of the above analysis remains valid even when the pole trajectory is reversed in Fig. 1(a) and (b), respectively. Also, if the original contour, C, is reversed in Fig. 1(a) and (b) so it is now the contour $C^{\prime} \equiv-C$, the results for $I(K)$ defined on $C^{\prime}$ are simply negative of those for $I(K)$ defined on $\mathrm{C}$.

\section{B. Uniform Behavior of VWM}

For the case in Fig. 1(a), when the pole is initially in the region $\operatorname{Im} \alpha_{p}>0$, once again (as in part A above), $\Lambda=-1$ and $U=1$ so from (26):

$$
\begin{aligned}
& \left.I^{V W M}(K)\right|_{\operatorname{Im} \alpha_{p}>0} \equiv I_{>}^{V W M}(K) \sim 2 \pi j\left(-R_{p}\right)+I_{S D P}^{N U}(K) \\
& +\frac{R_{p} e^{-j K a}}{\sqrt{-j a}} \sqrt{\frac{\pi}{K}}[1-F(-\sqrt{K a})]
\end{aligned},
$$

whereas, after the pole at $\alpha_{p}$ crosses the SDP and enters the region $\operatorname{Im} \alpha_{p}<0$, then $U=0$ in (26) so that

$$
\begin{aligned}
& \left.I^{V W M}(K)\right|_{\operatorname{Im} \alpha_{p}<0} \equiv I_{<}^{V W M}(K) \sim 0+I_{S D P}^{N U}(K)+ \\
& \frac{R_{p} e^{-j K a}}{\sqrt{-j a}} \sqrt{\frac{\pi}{K}}[1-F(+\sqrt{K a})]
\end{aligned}
$$

The continuity of VWM when $\alpha_{p}$ cross the SDP requires

$$
\lim _{\operatorname{Im} \alpha_{p} \rightarrow 0}\left[I_{>}^{V W M}(K)-I_{<}^{V W M}(K)\right]=0,
$$

or

$$
\lim _{\operatorname{Im} \alpha_{p} \rightarrow 0} \frac{R_{p} e^{-j K a}}{\sqrt{-j a}} \sqrt{\frac{\pi}{K}}[F(\sqrt{K a})-F(-\sqrt{K a})]=2 \pi j R_{p} .
$$

From (A-6), it is observed that (36) is true since

$$
\frac{R_{p} e^{-j K a}}{\sqrt{-j a}} \sqrt{\frac{\pi}{K}}\left[-2 \sqrt{\pi j K a} e^{j K a}\right]=2 \pi j R_{p} .
$$

Consequently (36) is indeed always satisfied whether or not $\alpha_{p}$ crosses the SDP through $\alpha \equiv \alpha_{s}=0$. Thus, the leading terms of $I^{V W M}(K)$ are always continuous and hence uniform. The above is also true for case (b) of Fig. 1.

\section{Relationship between PCM and VWM}

From (25) and (26) it is evident that one can express $I^{V W M}(K)$ in terms of $I^{P C M}(K)$ simply by noting that their difference is given by:

$$
I^{V W M}(K)-I^{P C M}(K) \equiv E(K),
$$

where

$$
E(K)=\left[I_{S D P}^{N U}(K)+\frac{R_{p} e^{-j K a}}{\sqrt{-j a}} \sqrt{\frac{\pi}{K}}\right](1-F( \pm \sqrt{K a})) .
$$

From (18) and $G(0)=-\alpha_{p} G(0)$ together with $f\left(\xi_{p}\right)-j a=f\left(\xi_{s}\right)$,

$E(K)=e^{K f\left(\xi_{s}\right)} \sqrt{\frac{\pi}{K}}\left[\frac{G_{a}\left(\alpha_{p}\right)-G_{a}(0)}{\alpha_{p}-0}\right](1-F( \pm \sqrt{K a})) ; \operatorname{Im} \alpha_{p}{ }_{>}^{<}$

In the problem of wedge diffraction of a CSB by a straight, perfectly conducting wedge which is solved in section IV, the following is true; namely, the factor $\left(G_{a}\left(\alpha_{p}\right)-G_{a}(0)\right)$ is small when $\alpha_{p}$ is in the neighborhood of the saddle point at $\alpha=0$, 
whereas $(1-F)$ is small as $\alpha_{p}$ moves far from $\alpha=0$ so that $|\sqrt{K a}|$ is large. Thus, the product of the two terms in the square brackets of (40) is always seen to be negligible even for $\alpha_{p}$ only moderately far from $\alpha=0$. The above is also borne out numerically where $E(K) \approx 0$ for this special case of CSB diffraction by a wedge; however, due to space limits these large number of numerical studies are not shown here. It is noted that for $\alpha_{p}$ near the saddle point ( $\alpha=0$ ), the term in the square brackets of (40) tends to behave like the derivative (or slope) of $G$.

\section{APPLICATION OF PCM AND VMM FOR ANALYZING THE CANONICAL PROBLEM OF CSB DIFFRACTION BY A WEDGE}

Consider a z-directed uniform line source located at $\vec{\rho}^{\prime}=\left(\rho^{\prime}, \phi^{\prime}\right)$ in real space which illuminates a PEC infinite straight wedge as illustrated in Fig. 2. The location of this line source in real space is next analytically continued into complex space via $\overline{\tilde{\rho}^{\prime}}=\bar{\rho}^{\prime}-j \bar{b}$ to be positioned at $\left(\widetilde{\rho}^{\prime}, \widetilde{\phi}^{\prime}\right)$ so that it generates a beam field with its beam axis oriented along $\hat{b}=\cos \phi_{b} \hat{x}+\sin \phi_{b} \hat{y}$ [12] with $\phi_{b}$ being a real angle. The tilde denotes a complex quantity. The CSB field propagates along complex ray paths [12]. These complex rays and complex parameters $\left(\widetilde{\rho}^{\prime}, \widetilde{\phi}^{\prime}\right)$ etc; are illustrated only symbolically in Fig. 3 and are not the actual ray paths. In the paraxial region, the $\mathrm{CSB}$ reduces to a Gaussian beam (GB) whose waist is proportional to $b=|\bar{b}|[12]$. It can be shown that the total electric field, $\bar{E}=\hat{z} E_{z}$, and the magnetic field, $\bar{H}=\hat{z} H_{z}$, associated with a CSB due to an electric and magnetic line source, respectively, at $\overrightarrow{\widetilde{\rho}}^{\prime}$ can be written in terms of a Green's function, $u_{s, h}(\rho, \tilde{\rho})$ as

$$
E_{z}\left(\rho, \widetilde{\rho}^{\prime}\right)=-j \omega \mu u_{s}\left(\rho, \widetilde{\rho}^{\prime}\right) ; H_{z}\left(\rho, \widetilde{\rho}^{\prime}\right)=-j \omega \varepsilon M u_{h}\left(\rho, \widetilde{\rho}^{\prime}\right)
$$

where the real observation location is at $\vec{\rho}=(\rho, \phi)$. Also, $\mu$ and $\varepsilon$ are the permeability and permittivity of the isotropic, homogeneous medium (e.g., free space) surrounding the wedge, respectively. In (41), $I$ and $M$ are the strengths of the electric and magnetic line sources, respectively. The subscripts 's' and ' $h$ ' on the wedge Green's function represent the cases for which the line source is 'electric' and 'magnetic', respectively.

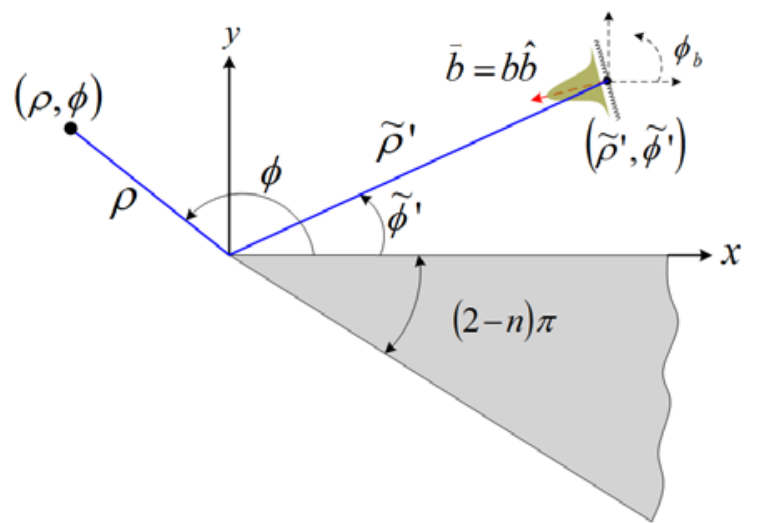

Fig. 2: Complex line source beam illumination of the wedge. The beam parameter is denoted by $\vec{b}$ and $\hat{b}$ is the direction of beam axis. Complex ray and source locations are drawn only symbolically; these are not actual ray paths which are complex.

The spectral integral representation of the Green's function $u_{s, h}(\rho, \widetilde{\rho})$ for $\mathrm{z}$ directed electric or magnetic line source excitation used here is the one given in [3] for the real source case (i.e. for a real source location); this exact result is analytically continued to deal with a line source in complex space, Thus,

$$
u_{s, h}\left(\rho, \widetilde{\rho}^{\prime}\right)=u\left(\rho, \widetilde{\rho}^{\prime} ; \widetilde{\beta}^{-}\right) \mp u\left(\rho, \widetilde{\rho}^{\prime} ; \widetilde{\beta}^{+}\right)
$$

where $\widetilde{\beta}^{\mp}=\phi \mp \widetilde{\phi}^{\prime}$, and for large $K$ with $K=k$ here,

$$
\begin{aligned}
& u\left(\rho, \widetilde{\rho}^{\prime} ; \widetilde{\beta}^{\mp}\right) \approx \frac{1}{8 \pi^{2} j n} \int_{L-L^{\prime}} d \xi\left(e^{-j k \gamma(\xi)} \sqrt{\frac{\pi}{2 j k \gamma(\xi)}} .\right. \\
& \left.. \cot \left(\frac{\xi+\widetilde{\beta}^{\mp}}{2 n}\right)\right) ; \quad \gamma(\xi)=\sqrt{\rho^{2}+\widetilde{\rho}^{\prime 2}-2 \rho \widetilde{\rho}^{\prime} \cos \xi}
\end{aligned}
$$

The exponential in (43) is approximated as by its two term binomial expansion as

$$
e^{-j k \gamma(\xi)} \approx e^{-j k\left(\rho+\widetilde{\rho}^{\prime}\right)} e^{j k \frac{\rho \widetilde{\rho}^{\prime}}{\rho+\widetilde{\rho}^{\prime}}(\cos \xi+1)}
$$

where $\frac{2 \rho \tilde{\rho}^{\prime}}{\left(\rho+\tilde{\rho}^{\prime}\right)^{2}}(\cos \xi+1)$ is assumed to be small; this approximation can be justified a posteriori. Therefore, incorporating (44) into (43) yields

$$
u\left(\rho, \widetilde{\rho}^{\prime} ; \widetilde{\beta}^{\mp}\right) \approx \int_{L-L^{\prime}} g\left(\xi, \widetilde{\beta}^{\mp}\right) e^{k f(\xi)} d \xi
$$

where

$$
g\left(\xi, \widetilde{\beta}^{\mp}\right)=\frac{1}{8 \pi^{2} j n} \sqrt{\frac{\pi}{2 j k \gamma(\xi)}} \cot \left(\frac{\xi+\widetilde{\beta}^{\mp}}{2 n}\right) e^{-j k\left(\rho+\widetilde{\rho}^{\prime}\right)}
$$

and

$$
f(\xi)=j \frac{\rho \rho^{\prime}}{\rho+\widetilde{\rho}^{\prime}}(1+\cos \xi)
$$

The saddle points of $f(\xi)$ are found via $\left.f^{\prime}(\xi)\right|_{\xi=\xi_{s}}=0$, and given by $\xi_{s}= \pm m \pi$ where $m=0,1,2,3, \cdots$. The $g\left(\xi, \widetilde{\beta}^{\mp}\right)$ in (46) also has branch point singularities at $\xi=\xi_{b}$ which are roots of $\rho^{2}+\widetilde{\rho}^{\prime 2}-2 \rho \widetilde{\rho}^{\prime} \cos \xi=0 \quad$ by $\quad \xi_{b}=2 \ell \pi \pm j \cosh ^{-1} \frac{\rho^{2}+\tilde{\rho}^{\prime 2}}{2 \rho \tilde{\rho}^{\prime}}$ $, \ell=0, \pm 1, \pm 2, \pm 3, \ldots$ and for $\rho$ and $\left|\tilde{\rho}^{\prime}\right|$ not too small, $\xi_{b}$ are not in the vicinity of the saddle points at $\xi_{s}= \pm m \pi$. In order to facilitate the Cauchy's theorem in evaluating the integral in (45), one has to determine SDPs to form a closed path with $C=L-L^{\prime}$. The SDPs are obtained by the conditions, $\operatorname{Im} f(\xi)=\operatorname{Im} f\left(\xi_{s}\right)$ and $\operatorname{Re} f(\xi)<0$ as $\operatorname{Im} \xi \rightarrow \infty$. Thus only SDPs that pass through the saddle point $\xi_{s}= \pm \pi$ need to be considered as shown in Fig. 3. The $g\left(\xi, \widetilde{\beta}^{\mp}\right)$ also has simple pole-type singularities at $\xi=\xi_{p}^{ \pm}=-\widetilde{\beta}+2 n N^{ \pm} \pi$, where $N^{ \pm}$determines the pole which is closest to one of saddle points. The superscript ' \pm ' on $\xi_{p}$ and $N$ pertains to the saddle point $\xi_{s}= \pm \pi . N^{ \pm}$is defined as the integer that most nearly satisfies the following 
equation.

$$
N^{ \pm}(\widetilde{\beta})=\frac{ \pm \pi+\operatorname{Re}(\widetilde{\beta})}{2 \pi n}
$$

The interior wedge angle is defined as $(2-n) \pi$ in Fig. 2. Therefore, the saddle points at $\xi_{s}= \pm \pi$, and only the poles at $\xi_{p}^{ \pm}=-\widetilde{\beta}+2 n N^{ \pm} \pi$ that lie within the closed path $C+S D P(\pi)+S D P(-\pi)$, respectively, contribute to the integral. It is noted that for sufficiently large $k$, the major contribution to the integrals evaluated over $\operatorname{SDP}( \pm \pi)$ occurs only from the "immediate vicinity" of the saddle points $\left(\xi_{s}= \pm \pi\right)$. Thus, $(\cos \xi+1)$ becomes very small as $\xi \rightarrow \xi_{s}= \pm \pi$, which is the sufficient condition for the approximation of (44). Therefore, the integral in (45) is deformed into SDPs via Cauchy's Theorem as follows

$$
\begin{aligned}
u\left(\rho, \tilde{\rho}^{\prime} ; \tilde{\beta}^{\mp}\right) \approx & -u_{S D P(\pi)}\left(\xi_{p}^{+}, \tilde{\beta}^{\mp}\right)+u_{P W}\left(\xi_{p}^{+}, \tilde{\beta}^{\mp}\right) U\left(\xi_{p}^{+}\right) \\
& -u_{S D P(-\pi)}\left(\xi_{p}^{-}, \tilde{\beta}^{\mp}\right)+u_{P W}\left(\xi_{p}^{-}, \tilde{\beta}^{\mp}\right) U\left(\xi_{p}^{-}\right)
\end{aligned}
$$

where

$$
u_{S D P( \pm \pi)}\left(\xi_{p}^{ \pm}, \widetilde{\beta}^{\mp}\right)=\int_{S D P( \pm \pi)} g\left(\xi, \beta^{\mp}\right) e^{k f(\xi)} d \xi
$$

and

$$
u_{P W}\left(\xi_{p}^{ \pm}, \beta^{\mp}\right)=2 \pi j R_{p}\left(\xi_{p}^{ \pm}, \widetilde{\beta}^{\mp}\right) .
$$

In (51), $R_{p}\left(\xi_{p}^{ \pm}, \widetilde{\beta}^{\mp}\right)$ is a residue of the integrand in (43) for corresponding poles. The residue contribution in (49) is automatically restricted by the Heaviside step function $U\left(\xi_{p}^{-}\right)$ to be associated with only those poles which lie within the closed path of integration formed by $C=L-L^{\prime}$ and $(S D P= \pm \pi)$. Therefore, the residue contributions represent the fields of an incident or reflected CSB produced by the complex line source which excites the wedge. The residue $R_{p}$ is found to be

$$
R_{p}\left(\xi_{p}^{ \pm}, \widetilde{\beta}^{\mp}\right)=\frac{1}{2 \pi j}\left\{\frac{-j}{4} \sqrt{\frac{2 j}{\pi k \gamma\left(\xi_{p}^{ \pm}\right)}} e^{-j k \gamma\left(\xi_{p}^{ \pm}\right)}\right\}
$$

$$
\operatorname{Im}(\xi)
$$

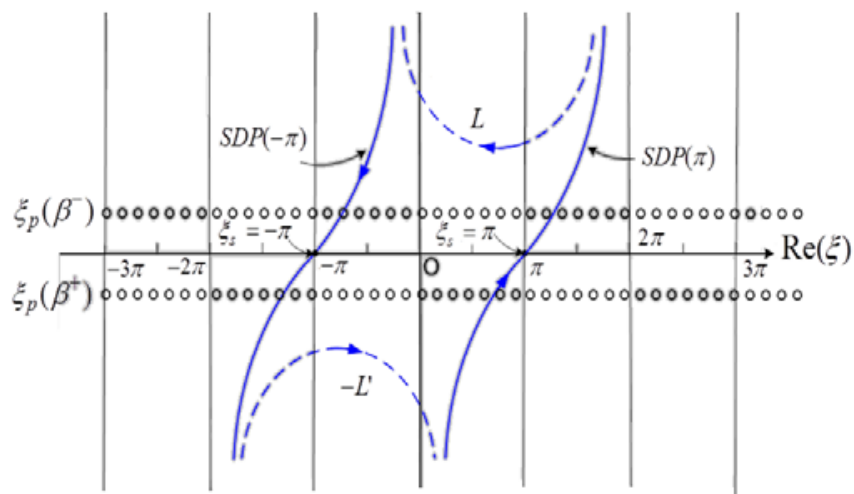

Fig. 3: Steepest descent paths, SDP $( \pm \pi)$ and the complex $\xi$ plane topology. The saddle points and poles are located at $\xi_{s}$ and $\xi_{p}\left(\operatorname{Im}\left(\tilde{\phi}^{\prime}\right)<0\right)$, respectively. The branch points $\xi_{b}$ are not enclosed by the closed contour and are far from $\xi_{\mathrm{s}}$. Thus, $\xi_{b}$ are not shown.

Therefore, incorporating (49) into (42) yields

$$
\begin{aligned}
u_{s, h}\left(\rho, \tilde{\rho}^{\prime}\right) \approx & \left\{-u_{S D P(\pi)}\left(\xi_{p}^{+}, \tilde{\beta}^{-}\right)+u_{P W}\left(\xi_{p}^{+}, \tilde{\beta}^{-}\right) U\left(\xi_{p}^{+}\right)\right. \\
& \left.-u_{S D P(-\pi)}\left(\xi_{p}^{-}, \tilde{\beta}^{-}\right)+u_{P W}\left(\xi_{p}^{-}, \tilde{\beta}^{-}\right) U\left(\xi_{p}^{-}\right)\right\} \\
\mp & \left\{-u_{S D P(\pi)}\left(\xi_{p}^{+}, \tilde{\beta}^{+}\right)+u_{P W}\left(\xi_{p}^{+}, \tilde{\beta}^{+}\right) U\left(\xi_{p}^{+}\right)\right. \\
& \left.-u_{S D P(-\pi)}\left(\xi_{p}^{-}, \tilde{\beta}^{+}\right)+u_{P W}\left(\xi_{p}^{-}, \tilde{\beta}^{+}\right) U\left(\xi_{p}^{-}\right)\right\}
\end{aligned}
$$

The first and the second residue terms $\left(u_{P W}\right.$ in(53)) contribute to the incident CSB electric/magnetic field for the n-face and oface illumination, respectively, whereas the third and the forth residue terms contribute to the reflected CSB electric/magnetic field arising from the $n$-face and o-face illumination, respectively, Since the total beam fields are composed of a superposition of the incident CSB, the reflected CSB, and beam diffracted fields, respectively, the saddle point contributions in (53) must thus correspond to the beam diffracted field, Using the solutions developed via PCM and VWM in the previous sections, one obtains the beam diffracted field as

$$
u_{S D P, s, h}^{\Gamma}\left(\rho, \rho^{\prime}\right) \approx u^{i} D_{s, h}^{\Gamma} \frac{e^{-j k \rho}}{\sqrt{\rho}} ; u^{i}=\frac{-j}{4} \sqrt{\frac{2 j}{\pi k}} \frac{e^{-j k \tilde{\rho}^{\prime}}}{\sqrt{\tilde{\rho}^{\prime}}}
$$

where $\Gamma$ denotes either PCM or VWM. The diffraction function terms $D_{s, h}^{\Gamma}$ in (54) for PCM and VWM are given by

$$
\begin{aligned}
& D_{s, h}^{P C M}=\frac{-e^{-j \pi / 4}}{2 n \sqrt{2 \pi k}}\left\{\left[\cot \left(\frac{\pi+\widetilde{\beta}^{-}}{2 n}\right) F\left( \pm \sqrt{k \widetilde{a}^{+}\left(\widetilde{\beta}^{-}\right)}\right)+\right.\right. \\
& \left.\cot \left(\frac{\pi-\widetilde{\beta}^{-}}{2 n}\right) F\left( \pm \sqrt{k \widetilde{a}^{-}\left(\widetilde{\beta}^{-}\right)}\right)\right] \mp\left[\cot \left(\frac{\pi+\widetilde{\beta}^{+}}{2 n}\right) .\right. \\
& \left.\left.F\left( \pm \sqrt{k \widetilde{a}^{+}\left(\widetilde{\beta}^{+}\right)}\right)+\cot \left(\frac{\pi-\widetilde{\beta}^{+}}{2 n}\right) F\left( \pm \sqrt{k \widetilde{a}^{-}\left(\widetilde{\beta}^{+}\right)}\right)\right]\right\}
\end{aligned}
$$

and

$$
\begin{aligned}
D_{s, h}^{V W M}= & {\left[d^{+}\left(\tilde{\beta}^{-}, \xi_{p}^{+}\left(\tilde{\beta}^{-}\right)\right)+d^{-}\left(\tilde{\beta}^{-}, \xi_{p}^{-}\left(\tilde{\beta}^{-}\right)\right)\right] } \\
& \mp\left[d^{+}\left(\tilde{\beta}^{+}, \xi_{p}^{+}\left(\tilde{\beta}^{+}\right)\right)+d^{-}\left(\tilde{\beta}^{+}, \xi_{p}^{-}\left(\tilde{\beta}^{+}\right)\right)\right],
\end{aligned}
$$

respectively, where

$$
\begin{aligned}
& d^{ \pm}\left(\widetilde{\beta}, \xi_{p}^{ \pm}(\widetilde{\beta})\right)=\frac{-e^{j \pi / 4}}{2 n \sqrt{2 \pi k}}\left\{\cot \left(\frac{\pi \pm \widetilde{\beta}}{2 n}\right) F\left( \pm \sqrt{k \widetilde{a}^{ \pm}(\widetilde{\beta})}\right)\right. \\
& +\left(-\sqrt{\frac{\rho+\widetilde{\rho}^{\prime}}{\gamma\left(\xi_{p}^{ \pm}(\widetilde{\beta})\right)}} \frac{n}{\cos \left(\xi_{p}^{ \pm}(\widetilde{\beta}) / 2\right)}+\cot \left(\frac{\pi+\widetilde{\beta}}{2 n}\right)\right) \\
& \left.\left(1-F\left( \pm \sqrt{k \widetilde{a}^{ \pm}(\widetilde{\beta})}\right)\right)\right\}
\end{aligned}
$$

and

$$
\widetilde{a}^{ \pm}(\beta)=\frac{2 \rho \widetilde{\rho}^{\prime}}{\rho+\widetilde{\rho}^{\prime}} \cos ^{2}\left(\frac{\xi_{p}^{ \pm}(\widetilde{\beta})}{2}\right)
$$

with $\gamma(\xi)$ being defined in (43). It is noted that $u^{i}$ represents the incident $\mathrm{CSB}$ at the edge for large $\left|k \widetilde{\rho}^{\prime}\right|$ where 
$u^{i}=-\frac{j}{4} H_{0}{ }^{(2)}\left(k \widetilde{\rho}^{\prime}\right)$. The $D_{s, h}^{P C M}$ and $D_{s, h}^{V W M}$ may be viewed as the CSB wedge diffraction coefficients found via PCM and VWM, respectively.

The reflected CSB appears to be emanate from the image of the actual complex source located at $\overline{\widetilde{\rho}}_{r}^{\prime}=\left(\widetilde{\rho}^{\prime},-\widetilde{\phi}^{\prime}\right)$. The incident $\left(E_{z}^{i}\right)$ and reflected $\left(E_{z}^{r}\right)$ CSB fields due to a z-directed complex electric line source and its image, respectively, are represented by their corresponding pole contributions and can be written as

$$
E_{z}^{i, r}(\bar{\rho})= \pm C_{0}\left(\frac{-j}{4} \sqrt{\frac{2 j}{\pi k}}\right) \frac{e^{-j k\left|\bar{\rho}^{-} \overline{\tilde{\rho}}_{i, r}^{\prime}\right|}}{\sqrt{\left|\bar{\rho}-\overline{\widetilde{\rho}}_{i, r}^{\prime}\right|}},
$$

where the negative sign for $E_{z}^{r}$ is due to the polarization change reversal arising from reflection off the PEC planar face. Also, $C_{0}=-j \omega \mu \mathrm{I}$ in (59) is a complex constant present in (41) As discussed in section II, the SBs for $E_{z}^{i, r}$ can be determined at points of observation for which the corresponding pole crosses the appropriate SDP. In other words, the SBs are found by numerically searching for the observation angle $\phi$ for which $\operatorname{Im} \alpha_{p}=0$ in the $\alpha$ plane. The $\alpha_{p}$ which allows one to find ISB and RSB are given, respectively, as

$$
\alpha_{p}^{i, r}\left(\widetilde{\beta}^{ \pm}\right)=-\sqrt{\frac{2 \rho \widetilde{\rho}^{\prime}}{\rho+\widetilde{\rho}^{\prime}}} \cos \left(\frac{\xi_{p}\left(\widetilde{\beta}^{\mp}\right)}{2}\right) e^{-j \frac{\pi}{4}},
$$

where the branch is specified as discussed in section II. It is noted that the computational effort to find ISB and RSB is negligible. The beam diffracted field can be found via PCM and VWM using (54)-(56), respectively, as

$$
E_{z}^{d, \Gamma}\left(\rho, \tilde{\rho}^{\prime}\right) \approx E_{z}^{i}\left(Q_{E}\right) D_{s, h}^{\Gamma} \frac{e^{-j k \rho}}{\sqrt{\rho}}
$$

where again $\Gamma=\mathrm{PCM}$ or VWM, $E_{z}^{i}\left(Q_{E}\right)$ is the incident CSB at the point of diffraction at $Q_{E}$ which is real for two dimensional case and located at the origin in this particular case, Therefore, the total CSB field at the presence of a wedge is obtained by the superposition of (59) and (61) as

$$
E_{z}^{\Gamma}\left(\rho, \tilde{\rho}^{\prime}\right) \approx E_{z}^{i}(\rho) U\left(\phi_{I S B}\right)+E_{z}^{r}(\rho) U\left(\phi_{R S B}\right)+E_{z}^{d, \Gamma}(\rho)
$$

with $\Gamma=\mathrm{PCM}$ or VWM, where $\phi_{I S B}$ and $\phi_{R S B}$ are the values of $\phi$ at ISB and RSB, respectively.

As an example, consider a line source located at $\left(\widetilde{\rho}^{\prime}, \widetilde{\phi}^{\prime}\right)$ or $\overline{\tilde{\rho}}^{\prime}=\bar{\rho}^{\prime}-j \bar{b}$ where $\bar{\rho}^{\prime} \rightarrow\left(\rho^{\prime}, \varphi^{\prime}\right)$ with $\rho^{\prime}=5 \lambda$ and $\phi^{\prime}=40^{\circ}$ in this example where $\lambda$ denotes the wavelength and $\phi_{b}=225^{\circ}$ which determines the direction of the beam axis. In this particular example, the beam axis hits the surface of wedge away from the edge. The beam parameter, $b=10 \lambda$. The real observation point is located at a distance $\rho=3 \lambda$ from the edge, and the observation angle $\phi$ covers all aspects external to the wedge. Finally, the incident, reflected, diffracted and total fields are computed from (59), (61) and (62) via both PCM and VWM as shown in Fig. 4. Note the fields are normalized by $C_{0} e^{k b}$ and $e^{k b}$ is the maximum value of $e^{-j k\left|\rho-\widetilde{\rho}^{\prime}\right|}$ in the $\hat{b}$ direction [12]. The total fields via both PCM and VWM are continuous across the ISB and RSB, and $E_{Z}^{P C M} \approx E_{Z}^{V W M}$. Another example is shown in Fig. 5. In this case, both $\rho$ and $\rho^{\prime}$ have been changed to $50 \lambda$ and $20 \lambda$, respectively. The other parameters are $b=20 \lambda, \phi^{\prime}=80^{\circ}$ and $\phi_{b}=258^{\circ}$. The wedge's angle is $W A=70^{\circ}$. The result in Fig. 5 again shows an excellent agreement between the two methods, i.e, $E_{z}^{P C M} \approx E_{z}^{V W M}$. Thus, for the CSB wedge diffraction solution found via PCM works surprisingly well because the PCM condition happens to be valid for this complex wave problem as verified by a large number of numerical studies, which cannot be presented here due space limitations. Also, one is referred to an analytical discussion below (40) which indicates the reason for $E(K) \approx 0$ in this special wedge problem.

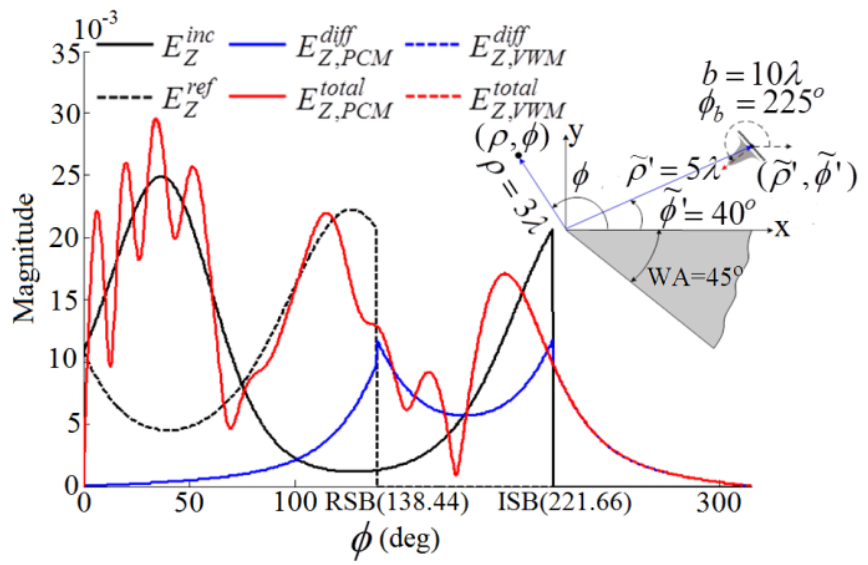

Fig. 4: Comparison of total field and diffracted field obtained by PCM and VWM approaches. The incident and reflected beams are shadowed at the incident SB (or ISB) and reflection SB (or RSB), respectively.

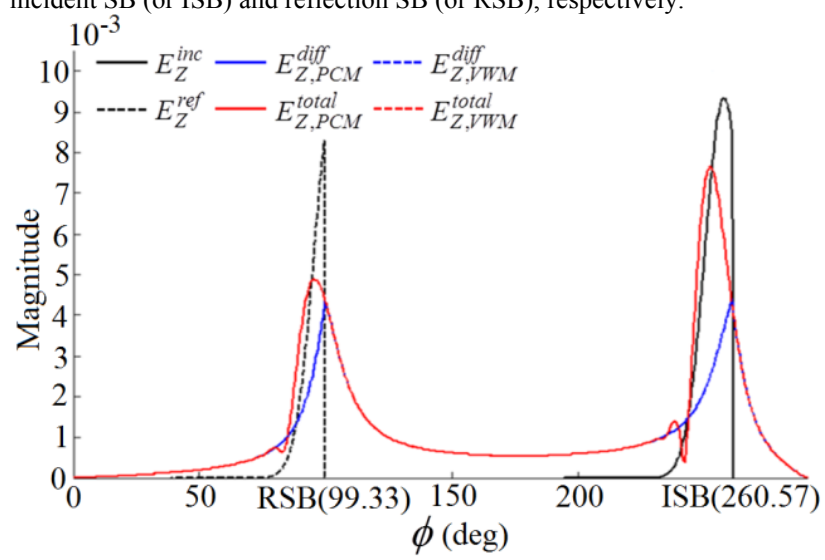

Fig. 5: Comparison of total field and diffracted field obtained by PCM and VWM approaches. The incident and reflected beams are shadowed at the incident SB (or ISB) and reflection SB (or RSB), respectively.

\section{CONCLUSION}

The validity of the leading terms of two uniform asymptotic methods, namely the PCM and VWM, is examined analytically and via applications. The PCM and VWM are useful for providing an analytical closed form solution to spectral wave integrals commonly occurring in EM radiation and diffraction problem.

Such wave integrals generally cannot otherwise be 
evaluated analytically in closed form. Initially, the leading terms of PCM and VWM are developed and summarized in a complete fashion, and in a user friendly form, for direct applications. The typical wave integrals considered are those whose integrands contain a first order saddle point and simple poles. The leading terms in the asymptotic high frequency regime are obtained via the usual SDP method. When the pole crosses the SDP the pole wave contribution becomes discontinuous since the pole goes from being captured to not being captured (or vice versa) in the deformation of the original contour of integration into the SDP. The uniform PCM and VWM contain a transition function which is supposed to provide a compensating discontinuity to keep the total solution composed of the pole wave and saddle point contribution continuous. However, it is shown that the simple PCM provide a continuous solution only if the PCM condition is met, while the VWM is always continuous without any restriction. For real waves, the PCM condition is usually satisfied, while for complex waves it is generally not satisfied. However, there are exceptions where the PCM condition can be satisfied even for complex waves, as is true for the problem of the CSB diffraction try a wedge which has been demonstrated here. On the other hand, the PCM condition is not met in the problem of excitation of complex waves such as surface and leaky waves on planar surfaces, and in this case only the VWM is valid. It is also shown that when the PCM condition is met, the difference between the VWM and PCM becomes negligible or zero as shown in the problem of CSB diffraction by a straight wedge in section IV. The latter result is highly significant as it provides a strong justification for obtaining a simple UTD type solution for the far more general problem of a diffraction of a CSB incident from an arbitrary direction on a wedge with arbitrary curvature directly by analytic continuation of a UTD result in [8] for a curved wedge excited by a point source in real space. Furthermore, the UTD result in [8] for the diffraction of an incident astigmatic real ray field by an arbitrary wedge cone likewise is analytically continued to treat the corresponding useful diffraction problem for an obliquely incident astigmatic GB.

\section{APPENDIX : ON THE TRANSITION FUNCTION $F( \pm \sqrt{K a})$}

The transition function $F( \pm \sqrt{K a})$, which occurs in the UTD solution is defined as

$$
F( \pm \sqrt{K a})=\sqrt{\frac{K}{\pi}} j a \int_{-\infty}^{\infty} \frac{e^{-K s^{2}}}{\alpha^{2}+j a} d \alpha
$$

From [2], (A-1) can be expressed in the more familiar form as [8]:

$$
\begin{gathered}
F( \pm \sqrt{K a})= \pm 2 j \sqrt{K a} e^{j \kappa a} \int_{ \pm \sqrt{K a}}^{\infty} e^{-j \tau^{2}} d \tau ; \\
{\left[\begin{array}{l}
-3 \pi / 4<\arg \sqrt{K a}<\pi / 4 \\
\pi / 4<\arg \sqrt{K a}<5 \pi / 4
\end{array} \quad(\mathrm{~A}-2)\right.}
\end{gathered}
$$

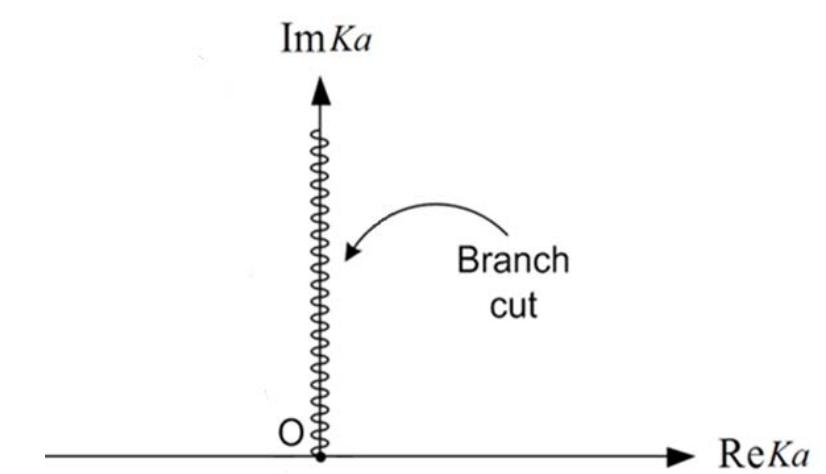

(a) Proper (or Top) Sheet in $K a$ plane exists where $-3 \pi / 2<\arg K a<\pi / 2$ Improper (or Bottom) Sheet in $\kappa a$ plane exists where $\pi / 2<\arg K a<5 \pi / 2$

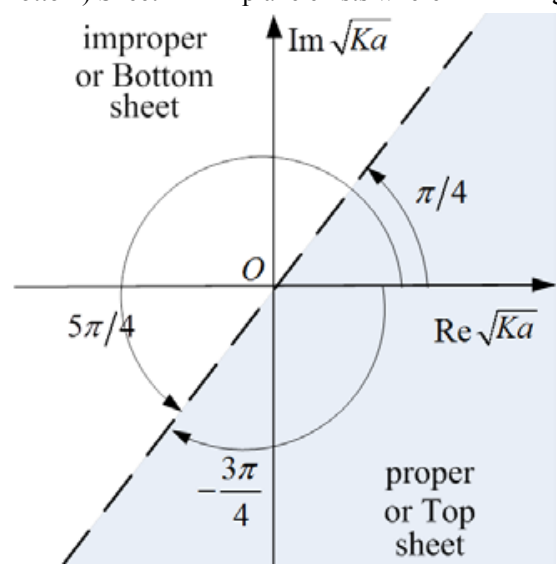

(b) Proper and Improper Sheets mapped onto the $\sqrt{K a}$ plane

Fig A.1: Branch cut for $F( \pm \sqrt{K a})$ to keep $F$ single valued and choose the proper sign on $\pm \sqrt{K a}$ so one always stays on the proper $\sqrt{K a}$ sheet, when the pole at $\alpha_{p}$ crosses the SDP (at $\operatorname{Im} \alpha=0$ ) by moving from $\operatorname{Im} \alpha_{p}>0$ to $\operatorname{Im} \alpha_{p}<0$ (or vice versa). On the proper sheet $F( \pm \sqrt{K a}) \rightarrow 1$ as $| \pm \sqrt{K a}| \rightarrow \infty$.

From Fig A.1(a) it follows that the proper sheet, or proper branch of $\sqrt{K a}$, is one for which $-3 \pi / 2<\arg K a<\pi / 2$, and the improper branch is one for which $\pi / 2<\arg K a<5 \pi / 2$. These two sheets are both mapped onto the $\sqrt{K a}$ plane in Fig. A.1 (b). Thus, if $\arg \sqrt{K a}$ lies on the proper sheet shown shaded in Fig. A.1 (b), then one must use $+\sqrt{K a}$,i.e. , use $F(+\sqrt{K a})$; however, if $\arg \sqrt{K a}$ lies on the improper sheet in Fig. A-1 (b), then one must replace $+\sqrt{K a}$ by $-\sqrt{K a}$ in $F$, i.e. one must use $F(-\sqrt{K a})$ so that one always stays on the proper branch. Now in [2],

$$
F_{C}( \pm \sqrt{K a}) \equiv e^{j K a} \int_{ \pm \sqrt{K a}}^{\infty} e^{-j \tau^{2}} d \tau .
$$

It follows from (A-2) and (A-3) that

$$
F( \pm \sqrt{K a})= \pm 2 j \sqrt{K a} F_{C}( \pm \sqrt{K a}) \text {. }
$$

Also, from [2]

$$
F_{C}(\sqrt{K a})+F_{C}(-\sqrt{K a})=\sqrt{\pi} e^{-j \pi / 4} e^{j K a} .
$$

Then from (A-4) and (A-5): 


$$
\begin{aligned}
F(+\sqrt{K a})-F(-\sqrt{K a}) & =2 j \sqrt{K a}\left[F_{C}(\sqrt{K a})+F_{C}(-\sqrt{K a})\right] \\
= & 2 \sqrt{\pi j K a} e^{j K a}
\end{aligned}
$$

Also, using [2], one can show that the large and small argument limits of $F( \pm \sqrt{K a})$ are:

$$
F( \pm \sqrt{K a}) \rightarrow 1 \text {, as }| \pm \sqrt{K a}| \rightarrow \infty
$$

and

$$
F( \pm \sqrt{K a}) \approx \pm \sqrt{\pi j K a} e^{j K a} \text {, as }| \pm \sqrt{K a}| \rightarrow 0
$$

\section{REFERENCES}

[1] P. C. Clemmow, "Some extensions to the method of integration by steepest descents," Quart. J Mech. Appl. Math. vol. 3, pp. 241-256,1950.

[2] P. C. Clemmow, "The Plane Wave Spectrum Representation of Electromagnetic fields." New York. Pergamon, 1966, pp. 56-58,

[3] P. H. Pathak and R.G. Kouyoumjian," The Dyadic Diffraction Coefficient for a Perfectly Conducting Wedge," Ohio State University, ElectroScience Lab., Tech. Rept. 2183-4, June 1970.

[4] T. B. A. Senior and J. L. Volakis, "Approximate Boundary Conditions in Electromagnetics," The Institute of Electrical Engineerings, London, 1995.

[5] E. L. Van der Waerden, "On the method of saddle points," Appl. Sci. Res., B2, pp. 3345, 1951.

[6] L. B. Felsen and N. Marcuvitz, "Radiation and Scattering of Waves," Englewood Cliffs, NJ: Prentice-Hall, 1973.

[7] R. E. Collin, "Field Theory of Guided Waves," IEEE Press Series on Electromagnetic Wave Theory, 1990

[8] R. G. Kouyoumjian and P. H. Pathak, "A uniform geometrical theory of diffraction for an edge in a perfectly conducting surface," Proc. IEEE, vol. 62 , pp. $1448-1461,1974$
[9] P. H. Pathak, "High Frequency Technique for Antenna Analysis," Proc. IEEE, Vol. 80, No. 1, pp. 44-65, Jan 1992.

[10] J. B. Keller and W. Strcifer, "Complex rays with an application to Gaussian beam," J. Opt. Soc. Amer., vol. 61, pp. 40-43, 1971.

[11] G. A. Deschamps, "Gaussian beam as a bundle of complex rays," Electron. Lett.,vol. 7, pp. 684-685, 1971.

[12] Felsen, "Complex source point solution of the field equations and their relation to the propagation and scattering of Gaussian Beams," Symposia Mathematica, vol. 18, pp. 39-56, 1976.

[13] R. G. Kouyoumjian, G. Manara, P. Nepa and B.J.E. Taute, "The diffraction of an inhomogeneous plane wave by a wedge," Radio Science, Vol. 31, Nov.-Dec., 1996.

[14] R. G. Kouyoumjian, T. Celandroni, G. Manara and P. Nepa, "Inhomogeneous electromagnetic plane wave diffraction by a perfectly electric conducting wedge at oblique incidence," Radio Science, vol. 42, Nov. 2007.

[15] C. Gennarelli and L. Palumbo, "A uniform asymptotic expansion of typical diffraction integrals with many coalescing simple pole singularities and a first-order saddle point," IEEE Trans. Antennas and Propagat., vol. AP-32, pp. 1122-1124, Oct. 1984.

[16] J. L. Volakis and M. 1. Herman, "A uniform asymptotic evaluation of integrals," Proc. IEEE, vol. 74, pp. 1043-1044, July 1986.

[17] E. L. Yip and R. J. Chiavetta, "Comparison of uniform asymptotic expansions of diffraction integrals," IEEE Trans. Antennas and Propagat., vol. 35 , no. 10 , Oct 1987 .

[18] R. G. Rojas, "Comparison between two asymptotic methods," IEEE Trans. Antennas and Propagation, vol. 35, no. 12, pp 1492-1493, Dec 1987.

[19] D. L. Hutchins and R. G. Kouyoumjian, "Asymptotic series describing the diffraction of a plane wave by a wedge," Ohio State Univ. ElectroSci. Lab., Techn. Rep. 2183-3, Dec. 1969.

[20] J. Boersma and Y. Rahmat-Samii, "Comparison of two leading uniform theories of edge diffraction with the exact nniform asymptotic solution," Radio Sci., vol. 15, pp. 1179-1194, Nov-Dec. 1980.

[21] M. Katsav, E. Heyman and L. Klinkenbusch, "Beam Diffraction by a Wedge: Exact and Complex Ray Solutions," in IEEE Trans. Antennas and Propagat., vol. 62, no. 7, pp. 3731-3740, July 2014. 